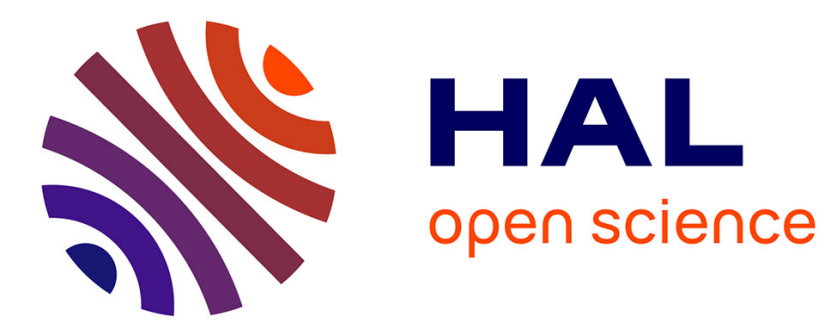

\title{
Very heavy fission fragments produced in the spallation reaction ${ }^{238} \mathrm{U}+\mathrm{p}$ at $1 \mathrm{~A} \mathrm{GeV}$
}

M. Bernas, P. Armbruster, J. Benlliure, A. Boudard, E. Casarejos, T. Enqvist, A. Kelic, R. Legrain, S. Leray, J. Pereira, et al.

\section{- To cite this version:}

M. Bernas, P. Armbruster, J. Benlliure, A. Boudard, E. Casarejos, et al.. Very heavy fission fragments produced in the spallation reaction ${ }^{238} \mathrm{U}+\mathrm{p}$ at $1 \mathrm{~A} \mathrm{GeV}$. Nuclear Physics A, 2006, 765, pp.197-210. 10.1016/j.nuclphysa.2005.10.009 . in2p3-00024371

\section{HAL Id: in2p3-00024371 https://hal.in2p3.fr/in2p3-00024371}

Submitted on 11 Oct 2005

HAL is a multi-disciplinary open access archive for the deposit and dissemination of scientific research documents, whether they are published or not. The documents may come from teaching and research institutions in France or abroad, or from public or private research centers.
L'archive ouverte pluridisciplinaire HAL, est destinée au dépôt et à la diffusion de documents scientifiques de niveau recherche, publiés ou non, émanant des établissements d'enseignement et de recherche français ou étrangers, des laboratoires publics ou privés. 


\title{
VERY HEAVY FISSION FRAGMENTS PRODUCED IN THE SPALLATION REACTION ${ }^{238} \mathrm{U}+\mathrm{p}$ at $1 \mathrm{~A} \mathrm{GeV}$.
}

\author{
M. Bernas ${ }^{a}$, P. Armbruster ${ }^{b}$, J. Benlliure $^{\mathrm{b}, \mathrm{c}}$, A. Boudard $^{\mathrm{d}}$, \\ E. Casarejos ${ }^{c}$, T. Enqvist ${ }^{\text {b }}{ }^{1}$, A. Kelic ${ }^{b}$, R. Legrain ${ }^{d}{ }^{2}$, \\ S. Leray ${ }^{\mathrm{d}}$, J. Pereira ${ }^{\mathrm{c}}$, F. Rejmund ${ }^{\mathrm{b}, \mathrm{a}}$, M.-V. Ricciardi ${ }^{\mathrm{b}}$, \\ K.-H. Schmidt ${ }^{\text {b }}$, C. Stéphan ${ }^{\text {a }}$, J. Taieb ${ }^{\text {b,a } 3}$, L. Tassan-Got ${ }^{\text {a }}$, \\ C. Volant ${ }^{\mathrm{d}}$. \\ ${ }^{a}$ Institut de Physique Nucléaire, 91406 Orsay Cedex, France \\ ${ }^{\mathrm{b}}$ Gesellschaft für Schwerionenforschung, Planckstr. 1, 64291 Darmstadt, Germany \\ ${ }^{c}$ Universidad de Santiago de Compostela, 15706 Santiago de Compostela, Spain \\ ${ }^{\mathrm{d} D A P N I A / S P h N, C E A / S a c l a y,} 91191$ Gif sur Yvette Cedex, France \\ ${ }^{\mathrm{e}}$ CEN Bordeaux-Gradignan, Le Haut-Vigneau, 33175 Gradignan Cedex, France
}

\begin{abstract}
Fission fragments from $1 \mathrm{~A} \mathrm{GeV}{ }^{238} \mathrm{U}$ ions impinging a hydrogen target are investigated by using the fragment separator FRS for magnetic selection of reaction products including ray-tracing, and $\Delta \mathrm{E}$-ToF techniques. On the basis of the kinematics of the fragments, binary fission can be assigned as production process up to very heavy fragments. Fission fragments are separated and identified as far as ${ }^{184}$ Re for the first time. Cross sections are measured and found in the range of $500 \mu \mathrm{b}$ to $5 \mu \mathrm{b}$. Isobaric cross sections decrease smoothly with increasing masses. Beyond tungsten, fission fragments are hidden in the more abundant spallation evaporation residues. 283 isotopes of elements gadolinium to rhenium in the mass range 147 to 184 are observed and cross sections measured. All the fragments are formed by fission of excited parent nuclei following emission of a cascade of secondary neutrons.
\end{abstract}

Key words: NUCLEAR REACTION p $\left({ }^{238} \mathrm{U}, \mathrm{x}\right), \mathrm{E}=1 \mathrm{GeV} /$ nucleon; Inverse kinematics method; In-flight separation with magnetic spectrometer; fission cross sections and velocities for 283 isotopes of elements from Gd up to Re. Relevance for ADS and radioactive beam production.

PACS: 24.75.+i: 25.40.Sc: 25.85.Ge: 28.50.Dr: 29.25.Rm

1 Present address: CUPP-project, P.O. Box 22 FIN-86801, Pyhäsalmi, Finland

2 deceased

3 Present address: CEA/Saclay DM2S/SERMA/LENR, Gif/Yvette, France

Preprint submitted to Nucl. Phys. A 


\section{Introduction}

The experimental study of ${ }^{238} \mathrm{U}$ fission induced by $1 \mathrm{GeV}$ protons has been performed by using inverse kinematics; the $1 \mathrm{~A} \mathrm{GeV} \mathrm{U}$ beam of the heavy-ion synchrotron (SIS) collides with the nuclei of a hydrogen target. Forward emitted fragments, bare from atomic electrons, are analysed in momentum with the high-resolution FRagment Separator FRS and fully identified in the associated detectors; time-of-flight (ToF) and energy loss of ions are measured. The velocity of each fragment is precisely determined and converted into the rest frame of the projectile. The velocity distribution for a given nuclide reveals the process from where it comes: either from fission of a more or less excited spallation residue leading to two fission fragments (FF), or from evaporation of nucleons leading to evaporation residues (EVR). The production cross sections are obtained by integrating the velocity distributions taking into account the transmission in the FRS. The main results from both reaction channels already published $[1,2]$ are briefly recalled. The total fission cross section for all elements between nitrogen and rhenium, $(1.53 \pm 0.15) \mathrm{b}$, is more than 3 times larger than the EVR-cross section, $(0.44 \pm 0.06)$ b.

EVR-production [2] is the main source of fragments close to U. After a rapid fall, within the 10 mass units close to $U$ covering $0.24 \mathrm{~b}$ - the main part of the EVR-cross section -, the mass-distribution decreases smoothly from $5 \mathrm{mb}$ to $4 \mathrm{mb}$ within a range of mass losses of 45 mass units. Finally a break-down by a factor of 5 to the level of $0.8 \mathrm{mb}$ is observed at a mass loss of 70 , where the EVR-cross sections equal the FF-cross sections, subject of this paper.

Fission leads mainly to fragments which cover the domain of $\mathrm{Z}=30$ to 60 . Their distribution of velocities and of isotopic yields are reported in [1]. Fragment velocities in the emitting-source frame and isotopic yields are shown to be consistent with a binary break-up of parent nuclei with $\mathrm{Z}_{0}=88$ to 92 . The shape of the measured velocity distributions reveals that even lightest fragments arise from a binary break-up [3]. The Z-distribution of the FF drops down from $\mathrm{Z}=45$ to $\mathrm{Z}=17$, forms a plateau until $\mathrm{Z}=14$ and increases again for smaller values of $Z$. The fission partners of these light elements are expected in the range $70 \leq \mathrm{Z} \leq 85$.

The present article reports on the first identification and measurement of very heavy fission products in the range of $\mathrm{Z}=64$ to 75 . We intend to bridge the regions of the nuclear chart populated by spallation [2] and by fission [1]. The $\mathrm{A}$ and $\mathrm{Z}$ limits between FF and EVR will be determined. Eventhough the fissioning parent nuclei are not directly accessible, the elemental and isotopic distribution of the heavy partners constrain the distribution of fissioning par- 
ents and should be reproduced by future models and simulations. The data complete the set of cross sections and kinetic energies for residues produced by fission and spallation for all elements between $\mathrm{Z}=7$ to $\mathrm{Z}=92$ in the reaction of $1 \mathrm{~A} \mathrm{GeV}^{238} \mathrm{U}+\mathrm{p}[1-3]$. A comprehensive overview of the results has been published recently [4]

\section{Experiment}

The uranium beam accelerated to an energy of $1 \mathrm{~A} \mathrm{GeV}(\mathrm{v} / \mathrm{c}=0.8)$ by the Heavy-Ion Synchrotron (SIS) at GSI-Darmstadt is focused on a thin liquid hydrogen target $\left(87 \mathrm{mg} \mathrm{cm}^{-2}\right.$ ) enclosed within Ti foils, at the bottom of a helium-cryostat [5]. In this velocity range, all fragments produced by the collision are forward emitted, which means that they are efficiently transferred into the $\pm 15 \mathrm{mrad}$ of angular acceptance of the FRS [6]. They are fully ionized $(q=Z)$ which makes safe their mass identification. The nuclear charge of each ion is obtained from the energy loss measured by the ionisation chamber placed at the exit of the FRS. The time of flight (ToF) is measured by two plastic scintillators [7], located in the intermediate dispersive and in the final focal plane respectively, separated by $37 \mathrm{~m}$. The magnetic field $B$ is set with an accuracy of $10^{-4}$. The radius of the trajectory $\rho$ is known from an accurate determination of the horizontal positions in the two scintillation detectors. The mass number $\mathrm{A}$ is calculated from the equation

$$
A=\frac{e}{m_{0} c} \frac{Z B \rho}{\beta \gamma}
$$

where $\mathrm{e}$ is the electron charge $\mathrm{c}$, the velocity of light and $\mathrm{m}_{0}$, the atomic mass unit. $\beta=\mathrm{v} / \mathrm{c}$ and $\gamma$, the Lorentz-factor, are derived from the time of flight (ToF).

A bunch of three to four isotopes of about 30 elements are simultaneously transferred within a momentum window $\Delta \mathrm{p} / \mathrm{p}=3 \%$ and registered. The longitudinal velocity distributions are reconstructed by combining the successive $\mathrm{B} \rho$ scannings normalized to the integrated U-beam current. For the present measurements the FRS setting was centered on $\operatorname{Re}(Z=75)$ in order to collect simultaneously fragments of Th down to Sm. The mass-number calibration is ensured by the reduced counting of the short-lived $\mathrm{N}=128$ isotopes, e.g. ${ }^{218} \mathrm{Th}$ which decays by $\alpha$-emission with a half-life $\mathrm{T}_{1 / 2}=0.1 \mu$ s before reaching the last FRS detectors.

Velocity distributions are transformed into the rest frame of the projectile. They are analysed to determine fission fragments velocities and production cross sections. In fission, the energy release from Coulomb repulsion between 

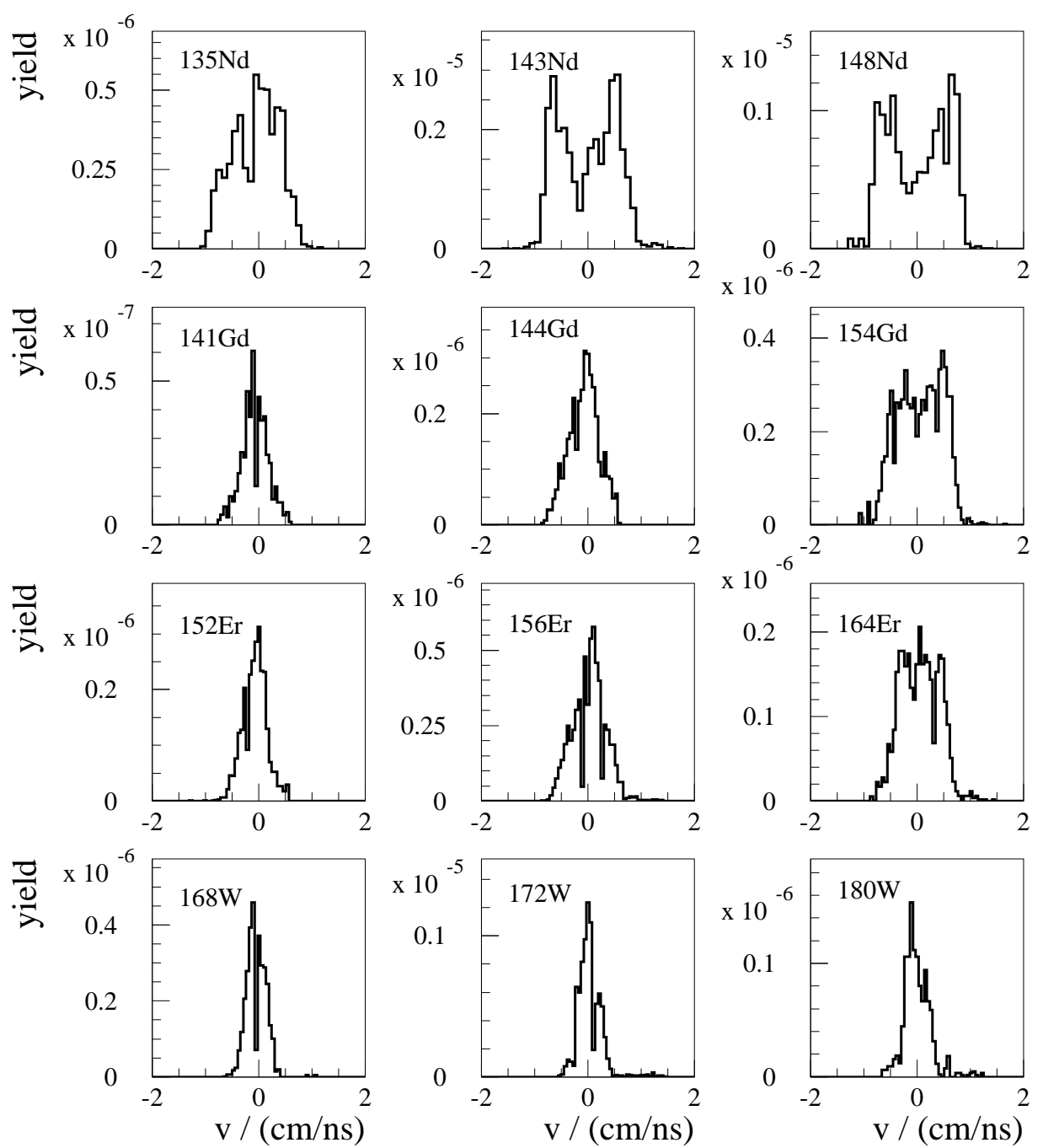

Fig. 1. Velocity distributions for selected isotopes in the beam rest frame of elements $\mathrm{Z}=64,68$ and 74. At the top line, data for isotopes of $\mathrm{Nd}(\mathrm{Z}=60)$, previously measured, illustrate the double peak shape of fission distributions.

the two emitted fragments is transferred into kinetic energy. The reconstructed velocity distribution of each fragment, a sphere in the frame of the fissioning nuclei, is truncated by the angular acceptance of the FRS. The velocity spectra of fission fragments show two peaks corresponding to the longitudinal projections of the parts transmitted forward and backward and the FFvelocity is calculated from these spectra. As an example velocity distributions of neodymium-isotopes are shown on the top row of Fig. 1. The transmission is calculated from the FF-velocity and the angular acceptance of the FRS of $\pm 15 \mathrm{mr}$ [1]. It reaches $100 \%$ for fragment-masses $\mathrm{A}>150$.

As the mass of FF increases, both peaks of forward and backward fission come close to each other, which reflects the decrease of velocity communicated to the heavy FF. They finally join into a rectangular distribution, as seen in Fig.1 
for ${ }^{154} \mathrm{Gd}$. For the isotopes of Gd, Er and $\mathrm{W}$ shown in Fig.1, the width of the velocity distributions decreases with the mass number. The three lightest isotopes of those elements, in the left column of Fig. 1, are EVR produced either in the Ti-windows of the $\mathrm{H}_{2}$ target or to a smaller amount, in secondary reactions of primary abundant heavier EVR [8,9]. The heaviest isotopes in the right column are fission fragments. The strong decrease of the width of the velocity distribution for $\mathrm{FF}$ going from $\mathrm{Gd}$ to $\mathrm{W}$ contrasts with the smaller decrease observed for EVR as seen in the left column. The isotopes shown in the central column are mixtures of EVR and FF with changing weights of both processes. The very different mass and charge dependencies of the velocity distributions for $\mathrm{FF}$ and $\mathrm{EVR}$ allow for a separation and an unfolding of the two production mechanisms $[2,10]$ as will be discussed in the following.

\section{Results}

\subsection{Separation of fission fragments}

The standard deviation of the velocity distributions of the fragments are taken as ingredients to evaluate fission velocities and the share of FF among the transmitted isotopes. In Fig. 2 the standard deviations are displayed as a function of the neutron number for isotopes of the heavy even- $Z$ elements. They remain constant for heavy isotopes as expected for FF and decrease linearly to a small constant value.

10 isotopes belong to the fission region for $\mathrm{Z}=68$ and 6 for $\mathrm{Z}=74$. Since fission rates from $\mathrm{Ti}$ window contribution have been measured to be only $3 \%$ of the rates for our target [1], 97\% of the FF are attributed to fission on hydrogen. For each element, the linear decrease of the width with decreasing neutron number follows the decrease of the contribution of fission relative to spallation-evaporation. Therefore, the relative weight of fission is evaluated by a linear interpolation between $100 \%$ of fission along the first part of the curve to $0 \%$ for the 3 to 5 lightest, most neutron-deficient fragments. For higher $\mathrm{Z}$, the contribution of fission diminishes and for osmium, $\mathrm{Z}=76$, the width is small and almost constant. Those fragments are produced by spallationevaporation. Two values of the width of EVR evaluated by J. Taieb [2] for $\Delta \mathrm{A}=50$ and 60 are reported on the plot to illustrate the coherence of both analysis. For the most neutron-deficient isotopes, the constant width of the velocity peak, shown in Fig. 2 by an horizontal dash for each element, decreases with increasing atomic number as expected for a pure EVR in agreement with the variation of the Morrissey systematics [11]. 


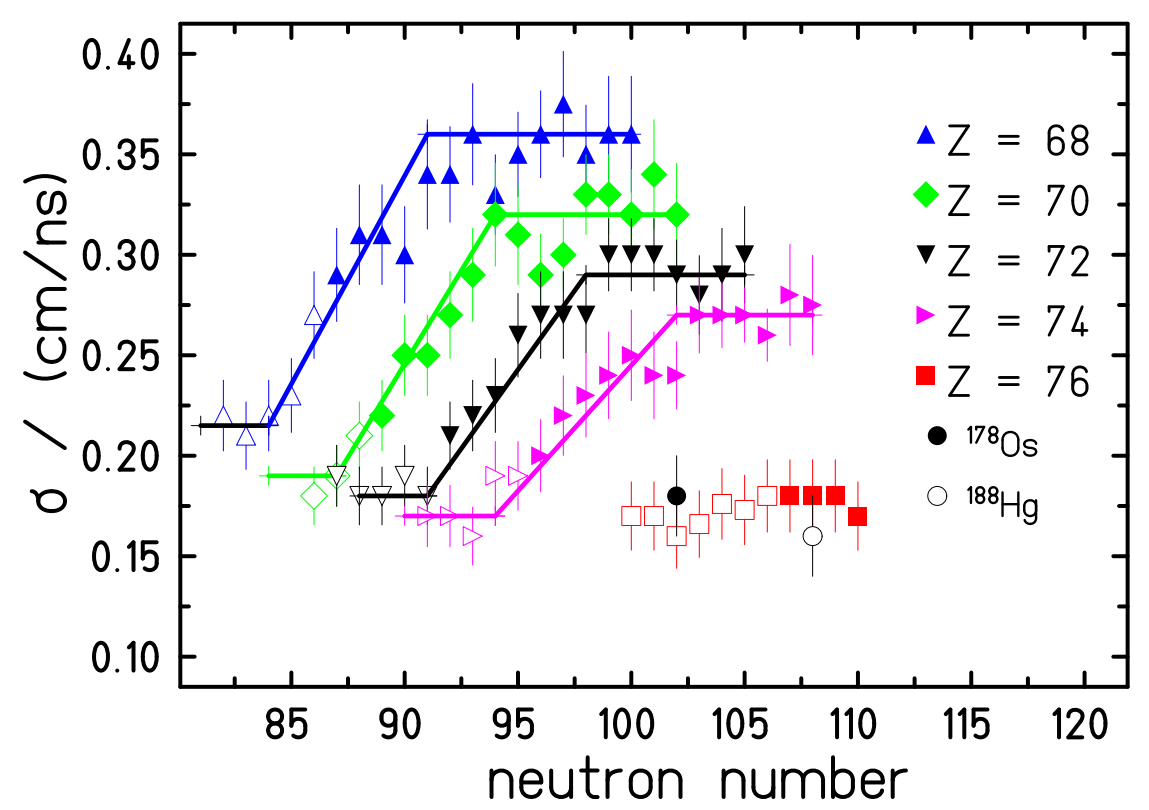

Fig. 2. Standard deviations of velocity distributions for nuclides produced as FF and as EVR. The horizontal parts of the lines indicate the standard deviations for FF (neutron-rich isotopes) and EVR (neutron-deficient isotopes). When the values drop down, the relative weight of fission diminishes versus evaporation. Empty symbols refer to isotopes where the contribution of fission was not extracted. The full and open circles shown on the curve for $\mathrm{Z}=76$ correspond to the widths measured for $\Delta \mathrm{A}=50\left({ }^{188} \mathrm{Hg}\right)$ and $60\left({ }^{178} \mathrm{Os}\right)$ respectively by J. Taieb [2].

\subsection{Velocities}

The FF-velocity $v_{f}$ is extracted from the measured shape of the velocity distribution. Assuming an isotropic spherical angular distribution for the emission of FF and a sharp value of the fission velocity, the longitudinal projection of the velocity vectors is expected to be a rectangle. The width of the rectangle (FWHM) is the sum of forward and backward velocities that is twice $v_{f}$.

The measured distribution is the convolution of this rectangle with a normal distribution (Gaussian shape) of the velocities of the selected FF, the standard deviation of which was evaluated to be $\sigma_{r}=0.13 \mathrm{~cm} / \mathrm{ns}$ in our previous work [1]. To this standard deviation contributes mainly the momentun spread of the fissioning parent nuclei, whereas the location straggling of the FF in the target is negligible; moreover, a fissioning species releases a total kinetic energy (TKE) showing a fluctuation in itself and there is an ensemble of different parent nuclei, each of them having a defined TKE. Together all fluctuations are transmitted to the FF and they determine the observed standard deviation $\sigma_{r}$ of $\mathrm{FF}$ velocity. The variance of the whole measured distribution $\sigma_{\text {meas }}^{2}$ is related to the variance of the rectangle $\sigma_{f}^{2}$ by $\sigma_{\text {meas }}^{2}=\sigma_{r}^{2}+\sigma_{f}^{2}$. As $\sigma_{f}$ is larger than $\sigma_{r}$, then $\sigma_{\text {meas }}^{2} \simeq \sigma_{f}^{2}=v_{f}^{2} / 3$, and it follows that $v_{f}=\sqrt{3} \sigma_{\text {meas }}$. The 


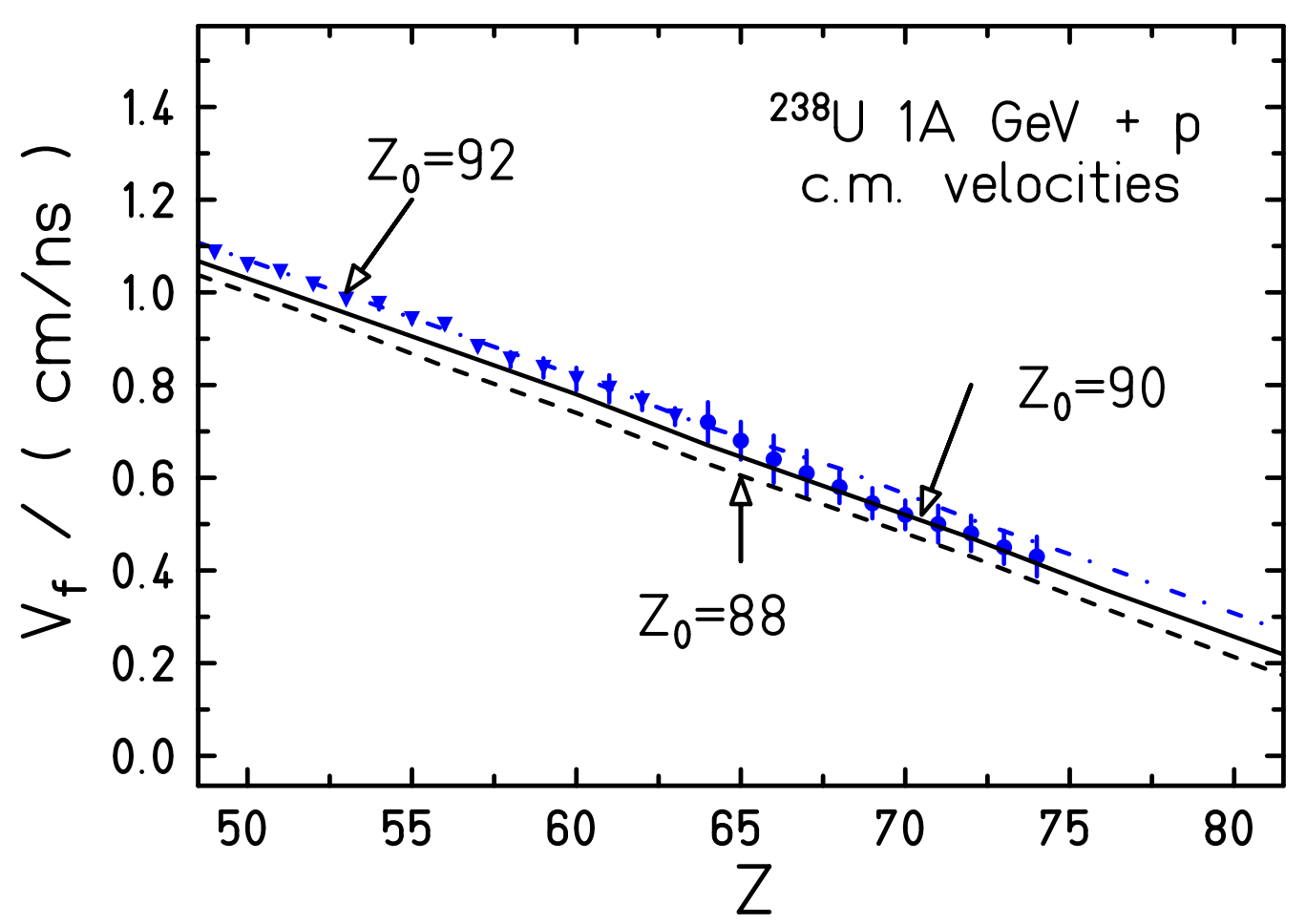

Fig. 3. Mean fission-fragment velocities in the rest frame as a function of the atomic number. The full points are from the present analysis and triangles from [1]. The curves are calculations for Ra, Th and $\mathrm{U}$ as parent nuclei.

velocities $v_{f}$ obtained for each $\mathrm{Z}$ are presented with full dots in Fig. 3. They complete coherently the curve obtained in the former evaluation for the main FF using a different method [1].

The total kinetic energy (TKE) released by fission is defined as the sum of the kinetic energies of the two FF, indexed 1 and 2. It equals the Coulomb energy between the fragments at scission. In eq. 1 the distance at scission is proportional to the sum of the radii of the two $\mathrm{FF}$, and is normalized by an effective radius constant $\mathrm{r}_{\text {eff }}$

$$
T K E=E_{1}+E_{2}=Z_{1} Z_{2} e^{2} / r_{e f f}\left(A_{1}^{1 / 3}+A_{2}^{1 / 3}\right)
$$

The velocity $v_{f}$ of a $\mathrm{FF}$ with mass and charge $\mathrm{A}_{1}, \mathrm{Z}_{1}$ produced in the fission of a parent nucleus $A_{0}, Z_{0}$ is calculated aplying the conservation of mass, charge and momentum at scission. Assuming isotropic emission of post-scission neutrons, the measured velocities after neutron emission are equal to the velocities of the primary fragments released from scission. The fission velocity is given by eq. 2 


$$
v_{f}\left(Z_{1}, A_{1}, Z_{0}, A_{0}\right) / c=Z_{2}\left[\frac{Z_{1} A_{2}}{A_{1} Z_{2}} \frac{2}{\left(A_{1}^{1 / 3}+A_{2}^{1 / 3}\right) A_{0}} \frac{e^{2}}{m_{0} c^{2} r_{e f f}}\right]^{1 / 2}
$$

The expression given is factorised into the $v_{f}^{\text {sym }}$, the velocity of the FF at symmetric fission and a factor giving the $\mathrm{Z}_{1}$ dependance of $v_{f}$ which is 1 for symmetric fission.

$$
v_{f}=v_{f}^{\text {sym }}\left[\frac{Z_{1} \cdot A_{2}}{Z_{2} \cdot A_{1}} \frac{2\left(A_{0} / 2\right)^{1 / 3}}{\left(A_{1}^{1 / 3}+A_{2}^{1 / 3}\right)}\right]^{1 / 2} \frac{Z_{2}}{\left(Z_{0} / 2\right)}
$$

with

$$
v_{f}^{s y m} / c=\frac{Z_{0}}{A_{0}^{2 / 3} 2^{5 / 6}}\left[\frac{e^{2}}{m_{0} c^{2} r_{e f f}}\right]^{1 / 2}
$$

A value of $v_{f}^{\text {sym }} / \mathrm{c}=4.0010^{-2}$ is calculated using ${ }^{220} \mathrm{Th}$ as the reconstructed mean fissioning parent nucleus [1] and $\mathrm{r}_{e f f}=1.86 \mathrm{fm}$. The value of $\mathrm{r}_{\text {eff }}$ is fixed demanding the TKE-values for symmetric fission of ${ }^{220} \mathrm{Th}$ following from eq.2 and from the TKE-model of Wilkins et al. [12] to become equal. For the high energy symmetric fission mode, we use the parameters proposed in [12]. Experimentally a value of $v_{f}^{s y m} / \mathrm{c}=3.8710^{-2}$ is found for ${ }^{107} \mathrm{Rh}$, the $\mathrm{FF}$ of symmetric fission. Using the latter value of $v_{f}^{s y m} / \mathrm{c}$, the measured value of $\overline{A_{1}}$, as presented in $[1,4]$ and the sum rules for mass and charge numbers giving $\mathrm{A}_{2}$ and $\mathrm{Z}_{2}$, we calculated the $\mathrm{Z}_{1}$ dependencies of the velocity shown in fig.3 for $\mathrm{Z}_{0}=88,90$, and 92 .

We obtain a remarkably good agreement between the measured values and the model presented. Taking the mean values of $\overline{A_{1}}$ determined for the light elements $\mathrm{Z}=12$ to $\mathrm{Z}=30$ [3], for the symmetric range [1] and the present values, all treated as described here, the velocity range covering $Z=12$ to $\mathrm{Z}=74$ was presented [4]. The $\mathrm{Z}_{1}$-dependance of $v_{f}$ shown in fig. 3 confirms that all elements investigated have parent nuclei, the majority of which is concentrated in a small range of atomic numbers $\mathrm{Z}_{0}=(90 \pm 2)$.

Using the mean mass value $\overline{A_{1}}$ of each element -weighted by their cross sections-, the mean kinetic energy per element is calculated from mean velocities. They are given in Table 1 .

\subsection{Isotopic cross sections}

The isotopic cross sections are calculated by integrating the center of mass velocity distributions of each isotope as sampled in Fig.1. A few corrections are required which were presented in detail in our previous papers [8,1]. 


\section{Table 1}

\begin{tabular}{|c|c||c|c||c|c|}
\hline $\mathrm{Z}$ & $\bar{E}_{k i n}[\mathrm{MeV}]$ & $\mathrm{Z}$ & $\bar{E}_{k i n}[\mathrm{MeV}]$ & $\mathrm{Z}$ & $\bar{E}_{k i n}[\mathrm{MeV}]$ \\
\hline 60 & $49.1 \pm 3.1$ & 65 & $36.7 \pm 4$. & 70 & $22.8 \pm 3.0$ \\
61 & $47.1 \pm 4.8$ & 66 & $33.0 \pm 4$. & 71 & $21.5 \pm 2.3$ \\
62 & $44.5 \pm 3.0$ & 67 & $30.3 \pm 3$. & 72 & $20.1 \pm 2.3$ \\
63 & $41.4 \pm 5$. & 68 & $27.8 \pm 3$. & 73 & $18.0 \pm 2.2$ \\
64 & $39.7 \pm 5$. & 69 & $24.9 \pm 3$. & 74 & $16.7 \pm 2$. \\
\hline
\end{tabular}

The mean kinetic-energy values of fission fragments as a function of the atomic number Z.

1) The transmission for fragments with low velocities is almost $100 \%$. For $64 \leq \mathrm{Z} \leq 67$, the fission velocity varies between 0.71 to $0.6 \mathrm{~cm} / \mathrm{hs}$ and the transmission increases from $90 \%$ to $100 \%$.(see Fig. $7 \mathrm{~b}$ in [1]). For Z> 67 the transmission is taken as $100 \%$.

2) Only totally stripped fragments $(q=Z)$ are analyzed here. However, at increasing atomic numbers the proportion of $\mathrm{H}$-like ionic states $(q=Z-1)$ is no longer negligible. For the present case, the data have to be multiplied by a factor increasing from 1.09 for $\mathrm{Z}=64$ to 1.23 for $\mathrm{Z}=73$ [13].

3) Another loss of FF comes from secondary reactions in the scintillator located at the dispersive focal plane in the middle of the FRS. This loss is calculated using the formulation of the total cross section from Karol [14]. The related correction factor is 1.17 for $64 \leq \mathrm{Z} \leq 75$.

4) The deviation of the mean trajectory for the isotopes of a given element from the optical axis also introduces a small loss. This correction factor, 1 for $\mathrm{Z}=74$ increases to 1.04 for $\mathrm{Z}=64$ [15], since the FRS setting was centered on $\operatorname{Re}(\mathrm{Z}=75)$.

5) The contribution of the dummy target (3\%) is substracted.

In Fig. 4 are collected the isotopic cross sections of the heavy FF for elements $\mathrm{Z}=64$ to $\mathrm{Z}=75$. Isotopic distributions show similar shapes for the elements investigated, and the maximum decreases smoothly from $0.4 \mathrm{mb}$ for gadolinium to $0.2 \mathrm{mb}$ for tungsten. The shares of EVR are calculated from the relative weights of the two mechanisms obtained from the width of velocity peaks as shown in Fig.2. The fission cross sections are given in table 2 with relative uncertainties. Systematic uncertainty reaches $10 \%$ as discussed in [1].

For the heaviest elements $\mathrm{Z}=70$ to 75 , the contribution of EVR is plotted with empty symbols. Secondary reactions taking place in the target remove a small 

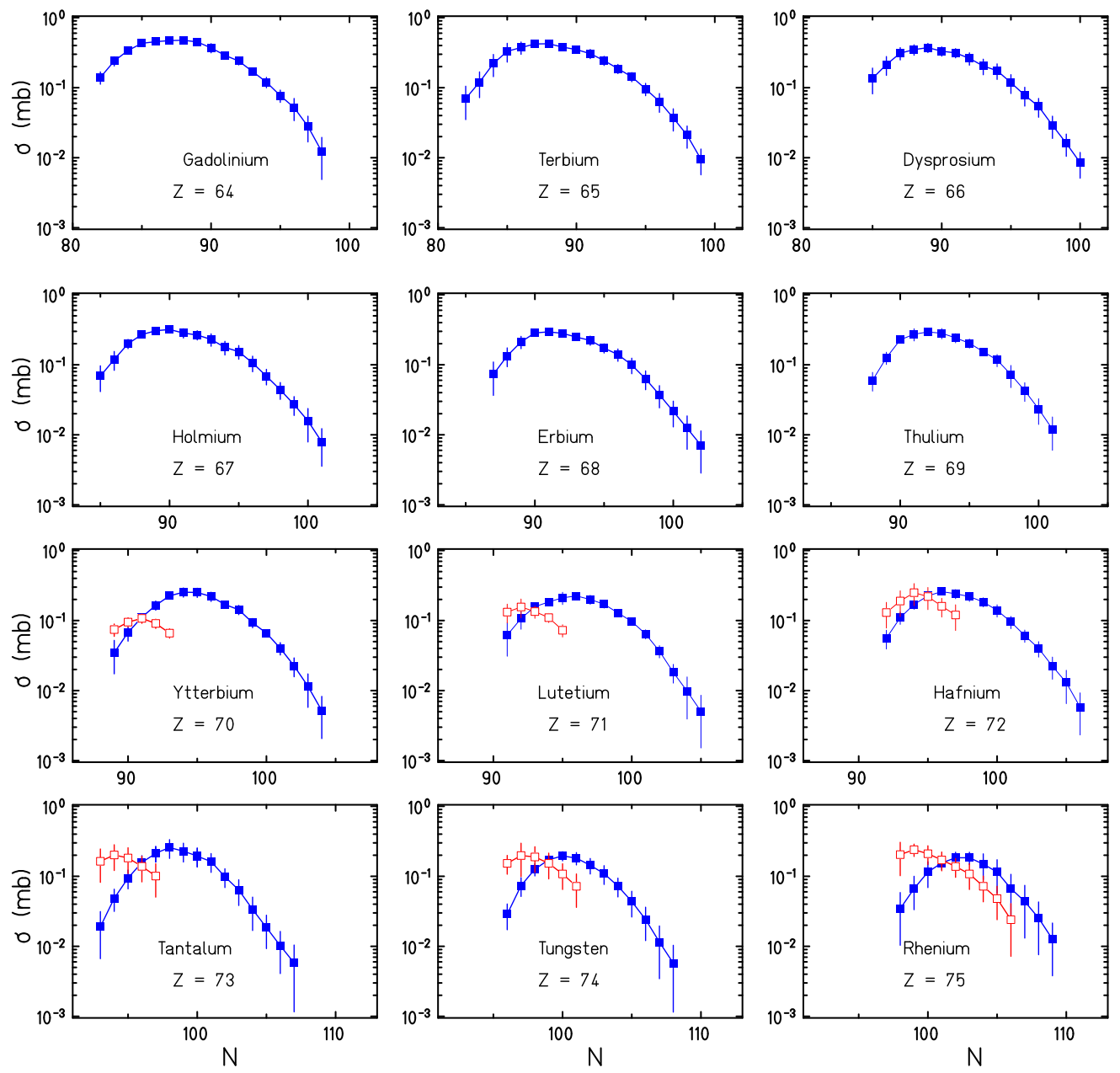

Fig. 4. Isotopic distributions of cross sections for elements gadolinium to rhenium. FF cross sections are represented by full symbols. The EVR contributions are shown with empty symbols for the heaviest elements. The large error bars reflect the uncertainties in the separation process.

fraction of FF towards lighter $\mathrm{Z}$ secondary products more neutron-deficient. Cross sections have been corrected for by using the method previously developed [9]. The width of the isotopic distributions decreases with increasing atomic numbers and it becomes more and more difficult to produce proton-rich nuclides. The present measurement of cross sections of FF is limited in the region of neutron-deficient isotopes because of major contributions due to EVR produced in the $\mathrm{Ti}$ windows and by secondary reactions. On the neutron-rich side, on the contrary, the selectivity of the FRS set-up allows to measure much smaller cross-sections. This was demonstrated earlier with the identification of 117 new FF in $\mathrm{U}(0.75 \mathrm{~A} \mathrm{GeV})+$ Be collisions [16]. However, in spite of a full transmission of the FRS for the heavy FF, the very weak statistics reflected by the large error bars restrict the measurement to cross sections larger than $5 \mu \mathrm{b}$. 


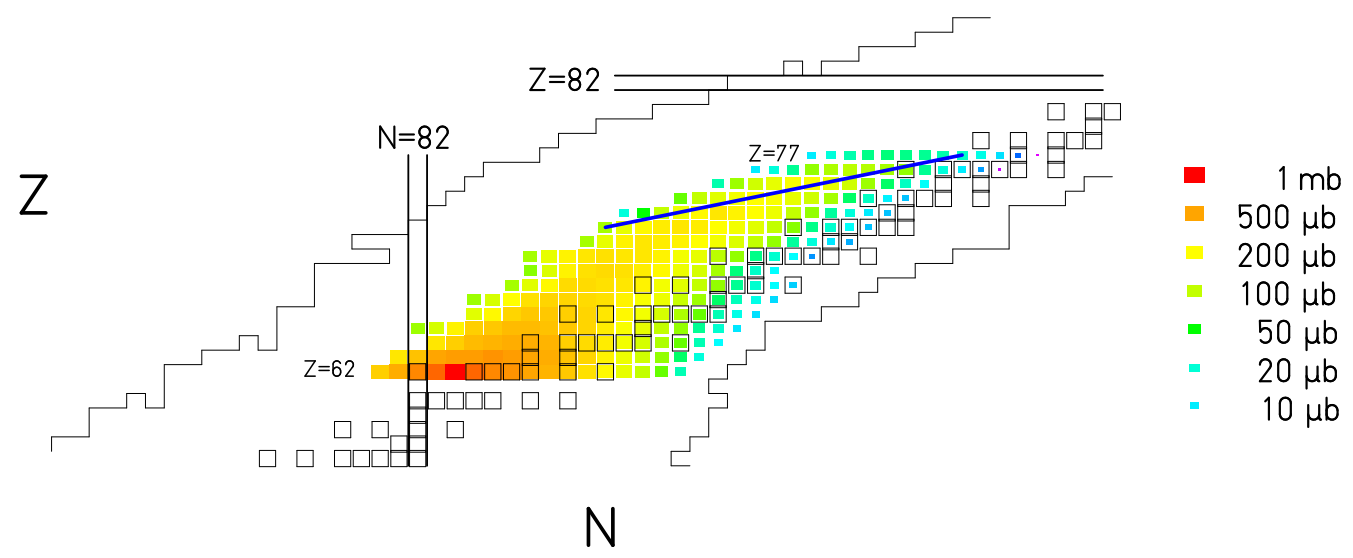

Fig. 5. Cluster-plot of the fission cross sections in the region where fission meets fragmentation. The line indicates equal production of FF and EVR. Extrapolated values are shown for the last 2 elements $\mathrm{Z}=76$ and 77

\section{Discussion}

The cluster-plot in Fig. 5 shows measured fission cross sections as a function of $\mathrm{N}$ and $\mathrm{Z}$ up to $\mathrm{Z}=75$. Equal production of $\mathrm{FF}$ and $\mathrm{EVR}$ is found for nuclides on the line shown in Fig. 5 which meets the line of maximum isotopic production at ${ }^{178}$ Re. Beyond the line of equal sharing, fission is still observable, as cross sections could be unfolded.

This division of cross sections between fission and evaporation populating the nuclear chart is a function of the excitation energy. In the reaction of $1 \mathrm{~A}$ $\mathrm{GeV}^{238} \mathrm{U}$ on deuterons, measured with the same set-up [10], the line of equal sharing between the two mechanisms is centered around $\mathrm{Z}=65,10$ elements lower. The region of the nuclear chart covered by EVR is larger and reaches smaller masses, near $\mathrm{A}=148$, since the mean energy transferred in the primary cascade for deuterons is doubled compared to protons. Beyond, in a collision of $1 \mathrm{~A} \mathrm{GeV}{ }^{238} \mathrm{U}$ on a slightly heavier target, all elements would be populated by EVR. We estimate a collision energy of 8 to $9 \mathrm{GeV}$ to reach this limit. The number of nuclides, about 1400 for ${ }^{238} \mathrm{U}$ on proton, could be increased by another 200 p-rich nuclides by using targets of ${ }^{9} \mathrm{Be}$ and beyond.

For ${ }^{238} \mathrm{U}+$ p, Fig. 6 shows that at $\mathrm{Z}=59$, where contributions of low-energy asymmetric fission mode disappear, the $\bar{N} / \mathrm{Z}$ ratio for heavy $\mathrm{FF}$ crosses the stability line. The FF are shifted to the proton rich side of the valley of stability. The last neutron-rich isotope shown in Fig. 5 is ${ }^{170} \mathrm{Tm}$.

In Fig. 7 the mass-distribution includes the range $\mathrm{A}_{2}=162-174$. A range of neutron numbers $\mathrm{N}_{2}=93-100$ and atomic numbers $\mathrm{Z}_{2}=69-74$ follows from the 
$\bar{N} / \mathrm{Z}=1.35$ measured for these elements (see Fig. 6). Isobaric cross sections drop from $1.1 \mathrm{mb}$ to $0.5 \mathrm{mb}$. To this range of cross sections correspond the masses $\mathrm{A}_{1}=45-36$, and the elements $\mathrm{Z}_{1}=20-16$ on the side of light FF. Their $\bar{N} / \mathrm{Z}$ ratios, measured and presented in $[3,4]$, cover a range from 1.25 to 1.22 , values much smaller than for their heavy partners.

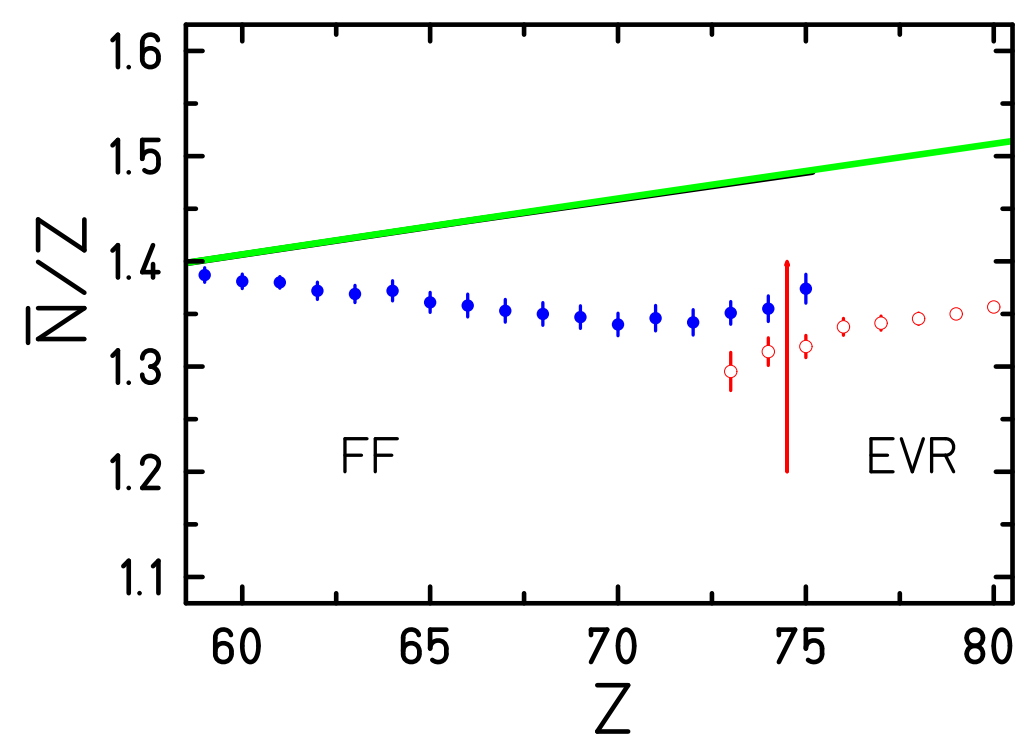

Fig. 6. Mean neutron number $\bar{N}$ divided by $\mathrm{Z}$ as a function of $\mathrm{Z}$ for fission (full symbols) and for fragmentation (empty symbols). The thick line shows the valley of stability and the vertical line, the separation of the regions populated by the two reactions.

The mass distribution of all fragments given in Fig. 7 is dominated by symmetric fission centered at $\mathrm{Z}=45, \mathrm{~A}=107$. Comparing the cross sections for light and heavy FF, a very small excess of cross sections for the light FF is observed. The distribution exibits asymmetric low-energy fission modes only by the two shoulders around $\mathrm{A}=132$ and $\mathrm{A}=96$. In the region of very light $\mathrm{FF}$, an enhancement of cross sections for decreasing masses is clearly seen for $A_{1}<32$ [3]. This behaviour, was observed before [17], and is explained by the Businaro-Gallone mountain in the liquid-drop potential energy surface and the unified fission model of Moretto $[18,19]$. A complementary increase of cross section for the heavy partner is expected, but it could not be observed here at the energy of $1 \mathrm{~A} \mathrm{GeV}$. Cross sections decrease continously up to $\mathrm{A}_{2}$ $=174$. Beyond it is very difficult to identify FF since they are more and more hidden by spallation-EVR. To find the increase of cross sections, the energy of $1 \mathrm{~A} \mathrm{GeV}$ should be lowered to $0.5 \mathrm{~A} \mathrm{GeV}$, and this phenomenon might be observed for masses larger than $\mathrm{A}_{2}=180$.

In our former work on symmetric fission [1] we separated the contribution of the low asymmetric fission modes from the high energy symmetric fission process. For the latter, the mean fission parent nucleus ${ }^{214} \mathrm{Th}$ was reconstructed from the mean pair of ${ }^{107} \mathrm{Rh}$ fission products. The attribution of $\mathrm{Z}=90$ to 
the mean parent nuclei was corroborated by the measured Z-dependence of the velocity distribution of $\mathrm{FF}$, assuming no protons to be evaporated and neutrons to be emitted isotropically. From the width of the $\mathrm{Z}$ distribution the mean excitation energy of $(58 \pm 10) \mathrm{MeV}$ was deduced [20]. It is converted into the emission of 6 neutrons from the two $\mathrm{FF}$ and a mean primary parent nucleus, ${ }^{220} \mathrm{Th}$, was restored.

A similar analysis is extended to very asymmetric splits assuming the same primary parent nucleus. This hypothesis is validated by the coherence of velocityvalues for very asymmetric FF with the previous curve as shown in Fig. 3. In case of a split in $\mathrm{Z}_{1} / \mathrm{Z}_{2}=16 / 74$, a value of $\overline{N_{1}} / \overline{N_{2}}=20 / 100$ is observed. The difference between the total number of 130 neutrons in the primary parent nucleus ${ }^{220} \mathrm{Th}$ and 120 neutrons in the observed asymmetric partners reaches 10. Very asymmetric fission releases 4 neutrons more than the mean split in symmetric fission. The corresponding excitation energy reaches $(90 \pm 15) \mathrm{MeV}$ compared to the mean value of $(58 \pm 10) \mathrm{MeV}$.

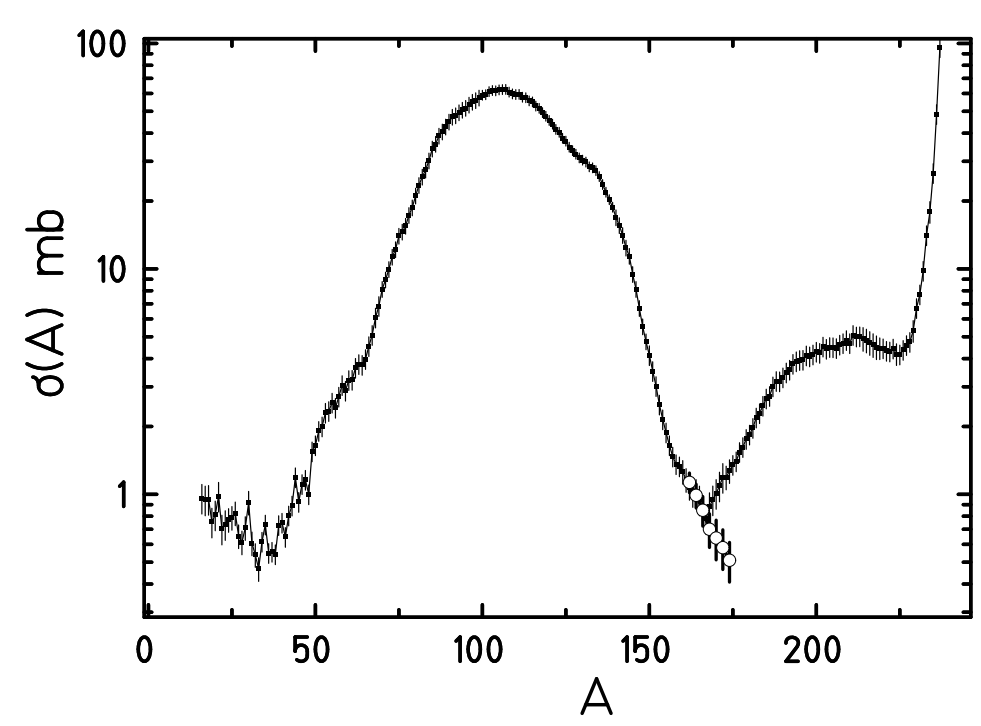

Fig. 7. Mass distribution of the production cross sections. The cross sections for FF are plotted as open circles for the mass range $A=162$ to 174 covered in this measurement.

The primary neutron numbers for the most asymmetric splits $Z_{1} / Z_{2}=16 / 74$, assuming still no proton evaporation, can be reconstructed making use of two principles applied to the formation of primary FF: the Unchanged-ChargeDensity (UCD) hypothesis and its correction for charge polarization. Taking ${ }^{220} \mathrm{Th}$ as primary parent nucleus and a division following the UCD hypothesis, the ratio of primary neutron numbers for $\mathrm{Z}_{1} / \mathrm{Z}_{2}=16 / 74$ would be $\mathrm{N}_{1} / \mathrm{N}_{2}=$ 23/107. Charge polarization, the difference between primary UCD-mass values and the actual primary mass values, makes light FF more neutron-deficient and heavy FF more neuron-rich as compared to UCD-masses. In high energy fission, neglecting nuclear structure in the FF, the difference is proportional 
to the mass asymmetry $\Delta$ of the mass split [21]. The charge polarization is $\delta \mathrm{A}=\left|\mathrm{A}_{2}-\mathrm{A}_{2}^{U C D}\right|=1 / 2 \mathrm{~A}_{0} \mathrm{P} \Delta=2.05$ a.m.u. , with the polarizability $\mathrm{P}$ $=2.89 .10^{-2}$ calculated from the potential energy surface, $\Delta$, the asymmetry of the mass split of our mass range and $\mathrm{A}_{0}=220$ from ${ }^{220} \mathrm{Th}$ as the mean parent nucleus. Taking into account the charge polarization correction, the ratio of primary neutron numbers is shifted to $21 / 109$. The neutrons emitted from each of the FF follows from the difference between the primary masses and the masses observed; 9 for the heavy FF and 1 for the light FF.

This sharing agrees with a division of the total energy in the fissioning system proportional to the sharing of the fragment mass relative to the total mass. For asymmetric mass splits, only a small part is found in the light FF whereas the bulk of energy is carried by the heavy FF. The latter deexcite by emission of a large number of neutrons, which explains their small value of $\bar{N} / \mathrm{Z}$ ratio.

We observed in the mass distribution, Fig. 7, as already stated, a small surplus of cross section for light FF which might disappear in the primary mass distribution. It is compensated by the dominant neutron emission from heavy FF, as well as by the charge polarization shifting neutrons into the heavy FF. The primary mass distribution is surprisingly symmetric and compatible with the fission of a few parent nuclei around ${ }^{220} \mathrm{Th}$. The majority of them fissions at excitation energies of about $60 \mathrm{MeV}$ and, as showed, only a few leading to very asymmetric splits fission at higher excitation energies, up to about 90 $\mathrm{MeV}$.

No indications for contributions of lighter fissioning parent nuclei were found, neither in the analysis of the cross section distribution, nor in the analysis of the velocity dependance of $\mathrm{FF}$ as a function of the atomic number, as shown in Fig. 3. They do exist [23] and might be established in a coming analysis using improved simulation codes for FF and EVR.

\section{Conclusion}

We have investigated the region of heavy FF populated by very asymmetric fission. Very few isotopes were separated before in this region, and there was no information on their kinematical properties. The $1 \mathrm{~A} \mathrm{GeV} \mathrm{U-beam} \mathrm{and}$ the high resolving power of the FRS provided the appropriate tools for this step ahead. Several nuclides in this region are of interest for activation analysis in technical applications of spallation reactions, e.g. in spallation-neutron sources, because they are long-lived alpha emitters.

For the first time, each isotopic cross section was measured and the process from where fragments originate was deduced from their velocity-distribution. Heavy FF result from a very asymmetric fission of hot parent nuclei. The exci- 
tation energy is released mainly by neutron emission from heavy FF which are shifted to the proton-rich side of the stability valley. The highly asymmetric splits do not lead to neutron-rich nuclides. For proton-rich nuclides, the production rates also break down, as for increasing atomic numbers the widths of the isotopic distributions decrease rapidly. This very asymmetric fission is not competitive to produce neither neutron-rich nor proton-rich nuclides. Here low energy fusion and cold fragmentation [22] are better suited for production of exotic nuclei.

Finally, the results presented here and in [1,3] illustrate a reaction close to the fission as imagined by the pioneers of our field in its first decade : the symmetric fission of a liquid drop ignoring nuclear structure.

Financial support by EU-contract ERBCHBCT940717 for J. B., T. E. and F. R. and by GSI for M. V. R. and J. T. is gratefully acknowledged. This work was partially supported by the European Union in the HINDAS project (contract FIKW-CT-20000-00031), by the support to the access to large facilities (contract EC-HPRI-CT-1999-00001.) and by the french-german collaboration agreement IN2P3-DSM/CEA and GSI.

\section{References}

[1] M. Bernas, P. Armbruster, J. Benlliure, A. Boudard, E. Casajeros, S. Czajkowski, T. Enqvist, R. Legrain, S. Leray, B. Mustapha, P. Napolitani, J. Pereira, F. Rejmund, M.-V. Ricciardi, K.-H. Schmidt, C. Stéphan, J. Taieb, L. Tassan-Got and C. Volant, Nucl. Phys. A725 (2003) 213

[2] J. Taieb, P. Armbruster, J. Benlliure, M. Bernas, A. Boudard, E. Casajeros, S. Czajkowski, T. Enqvist, R. Legrain, S. Leray, B. Mustapha, M. Pravikoff, F. Rejmund, K.-H. Schmidt, C. Stéphan, L. Tassan-Got, C. Volant and W. Wlazlo. Nucl. Phys. A724 (2003) 413 and Thesis IPN-Orsay, Univ. Paris XI, (2000).

[3] M.-V. Ricciardi Thesis, Santiago di Compostela (2005) and to be submitted to Nucl. Phys. A

[4] P. Armbruster, J. Benlliure, M. Bernas, A. Boudard, E. Casajeros, S. Czajkowski, T. Enqvist, S. Leray, P. Napolitani, J. Pereira, F. Rejmund, M.-V. Ricciardi, K.-H. Schmidt, C. Stéphan, J. Taieb, L. Tassan-Got and C. Volant, Phys. Rev. Lett. 93(21) 212701 (2004)

[5] P. Chesny, A. Forges, J. M. Gheller, G. Guiller, P. Pariset, L. Tassan-Got, P. Armbruster, K.-H. Behr, J. Benlliure, K. Burkard, A. Brünle, T. Enqvist, F. Fargett and K.-H. Schmidt, GSI-Ann. Rep. 1996, GSI 1997-1, p. 190

[6] H. Geissel, K.-H. Behr, A. Brünle, K. Burkard, M. Chen, H. Folger, B. Franczak, H. Keller, O. Klepper, B. Langenbeck, F. Nickel, E. Pfeng, M. Pfützner, E. Roeckl, K. Rykaczewski, I. Schall, C. Scheidenberger, K.-H. Schmidt, A. 
Schröter, T. Schwab, K. Sümmerer, M. Weber, G. Münzenberg, T. Brohm, H.-G. Clerc, M. Fauerbach, J.-J. Gaimard, A. Grewe, E. Hanelt, B. Knödler, M. Steiner, B. Voss, J. Weckenmann, C. Ziegler, A. Magel, H. Wollnik, J.-P. Dufour, Y. Fujita, D.J. Vieira and B. Sherill, Nucl. Inst. and Methods B 70 (1992) 286

[7] B. Voss, T. Brohm, H.-G. Clerc, A. Grewe, E. Hanelt, A. Heinz, A. Junghans, W. Morawek, C. Röhl, S. Steinhäuser, C. Zeigler, K.-H. Schmidt, K.-H. Behr, H. Geissel, F. Nickel, C.Sheidenberger, K. Sümmerer and A. Magel, Nucl. Instrum. and Methods A 364 (1995) 150

[8] T. Enqvist, P. Armbruster, J. Benlliure, M. Bernas, A. Boudard, S. Czajkowski, R. Legrain, S. Leray, B. Mustapha, M. Pravikoff, F. Rejmund, K.-H. Schmidt, C. Stéphan, J. Taieb, L. Tassan-Got, F. Vivés and C. Volant, Nucl. Phys. A 703 (2002) 435.

[9] P. Napolitani, P. Armbruster, M. Bernas and L. Tassan-Got, Nucl. Phys. A 727 (2003) 120

[10] E. Casajeros. Thesis, Santiago de Compostela (April 2002) and to be published in Phys. Rev. C

[11] D. J. Morrissey, Phys. Rev. C 39 (1989) 460

[12] B. D. Wilkins, E. P. Steinberg and R. R. Chasman, Phys. Rev C 14 (1976) 1832

[13] C. Scheidenberger, Th. Stöhlker, W.E. Meyerhof, H. Geissel, P.H. Mokler and B. Blank Nucl. Inst. and Methods 142 (1998) 441

[14] P. J. Karol Phys. Rev. C 11 (1975) 1203

[15] J. Pereira-Conca, Thesis, Santiago de Compostela (2004) and to be submitted to Phys. Rev. C

[16] M. Bernas, C. Engelmann, P. Armbruster, S. Czajkowski, F. Ameil, C. Böckstiegel, Ph. Dessagne, C. Donzaud, H. Geissel, A. Heinz, Z. Janas, C. Kozhuharov, C. Miehé, G. Münzenberg, M. Pfützner, W. Schwab, C. Stéphan, K. Sümmerer, L. Tassan-Got and B. Voss, Phys. Lett. B 415 (1997) 111

[17] D. G. Sarantites, D. R. Bowman, G. J. Wozniak, R. J. Charity, Z. H. Liu, R. J. McDonald, M. A. McMallan and L. G. Moretto, Phys. Lett. B218 (1989) 427

[18] U. Businaro and S. Gallone, Nuovo cimento 1, 1277 (1955)

[19] L. G. Moretto, Nucl. Phys. A 247 (1975) 211

[20] A. Ya. Rusanov, M. G. Itkis and V. N. Okolovich, Phys. At. Nucl. 60 (1997) 683

[21] P. Armbruster, Nucl. Phys. A 140 (1970) 385

[22] E. Hanelt, A. Grewe, K.-H. Schmidt, T. Brohm, H.-G. Clerc, M. Dornik, M. Fauerbach, H. Geissel, A. Magel, G. Münzenberg, f. Nickel, M. Pfützner, c. Scheidenberger, M. Steiner, K. Sümmerer, B. Voss,M. Weber, j. Weckenmann, C. Zeigler, Z. Phys. A 346 (1993) 43 
[23] B. Jurado, Thesis Santiago de Compostela (2003) and B. Jurado, C. Schmitt, K.-H. Schmidt, J. Benlliure, T. Enqvist, A. R. Junghans, A. Kelic and F. Rejmund, Phys. Rev. Lett. 93 (7)(2004) 072501 


\begin{tabular}{|c|c|c|}
\hline Z & A & $\sigma[\mathrm{mb}]$ \\
\hline \multirow[t]{16}{*}{64} & 147 & $0.26(4)$ \\
\hline & 148 & $0.34(4)$ \\
\hline & 149 & $0.429(34)$ \\
\hline & 150 & $0.454(45)$ \\
\hline & 151 & $0.47(12)$ \\
\hline & 152 & $0.476(24)$ \\
\hline & 153 & $0.45(5)$ \\
\hline & 154 & $0.34(5)$ \\
\hline & 155 & $0.29(3)$ \\
\hline & 156 & $0.24(3)$ \\
\hline & 157 & $0.17(2)$ \\
\hline & 158 & $0.12(2)$ \\
\hline & 159 & $0.077(14)$ \\
\hline & 160 & $0.052(7)$ \\
\hline & 161 & $0.028(5)$ \\
\hline & 162 & $0.012(7)$ \\
\hline \multirow[t]{12}{*}{65} & 147 & $0.07(4)$ \\
\hline & 148 & $0.12(5)$ \\
\hline & 149 & $0.221(77)$ \\
\hline & 150 & $0.331(99)$ \\
\hline & 151 & $0.374(75)$ \\
\hline & 152 & $0.421(42)$ \\
\hline & 153 & $0.424(42)$ \\
\hline & 154 & $0.38(4)$ \\
\hline & 155 & $0.35(5)$ \\
\hline & 156 & $0.306(46)$ \\
\hline & 157 & $0.244(36)$ \\
\hline & 158 & $0.187(28)$ \\
\hline
\end{tabular}




\begin{tabular}{|c|c|c|}
\hline & $0.143(21)$ \\
\hline & 160 & $0.096(19)$ \\
\hline & 161 & $0.063(19)$ \\
\hline & 162 & $0.037(12)$ \\
\hline & 163 & $0.021(7)$ \\
\hline & 164 & $0.0095(38)$ \\
\hline 66 & 151 & $0.136(54)$ \\
\hline & 152 & $0.214(64)$ \\
\hline & 153 & $0.311(62)$ \\
\hline & 154 & $0.35(6)$ \\
\hline & 155 & $0.373(56)$ \\
\hline & 156 & $0.332(50)$ \\
\hline & 157 & $0.314(47)$ \\
\hline & 158 & $0.262(52)$ \\
\hline & 159 & $0.204(51)$ \\
\hline & 160 & $0.175(44)$ \\
\hline & 161 & $0.118(35)$ \\
\hline & 162 & $0.078(23)$ \\
\hline & 163 & $0.054(16)$ \\
\hline & 164 & $0.029(10)$ \\
\hline & 165 & $0.016(6)$ \\
\hline & 166 & $0.0085(34)$ \\
\hline 67 & 152 & $0.069(30)$ \\
\hline & 153 & $0.119(36)$ \\
\hline & 154 & $0.20(3)$ \\
\hline & 155 & $0.27(3)$ \\
\hline & 156 & $0.306(31)$ \\
\hline & 157 & $0.319(32)$ \\
\hline & 158 & $0.284((43)$ \\
\hline & 159 & $0.266(40)$ \\
\hline
\end{tabular}




\begin{tabular}{|c|c|c|}
\hline \multicolumn{2}{|r|}{160} & $0.23(5)$ \\
\hline & 161 & $0.177(39)$ \\
\hline & 162 & $0.154(34)$ \\
\hline & 163 & $0.106(26)$ \\
\hline & 164 & $0.069(17)$ \\
\hline & 165 & $0.044(12)$ \\
\hline & 166 & $0.027(8)$ \\
\hline & 167 & $0.016(8)$ \\
\hline & 168 & $0.008(4)$ \\
\hline 68 & 155 & $0.073(36)$ \\
\hline & 156 & $0.134(40)$ \\
\hline & 157 & $0.212(42)$ \\
\hline & 158 & $0.285(28)$ \\
\hline & 159 & $0.295(29)$ \\
\hline & 160 & $0.281(28)$ \\
\hline & 161 & $0.248(25)$ \\
\hline & 162 & $0.223(33)$ \\
\hline & 163 & $0.173(26)$ \\
\hline & 164 & $0.14(3)$ \\
\hline & 165 & $0.099(25)$ \\
\hline & 166 & $0.063(19)$ \\
\hline & 167 & $0.037(13)$ \\
\hline & 168 & $0.022(9)$ \\
\hline & 169 & $0.012(6)$ \\
\hline & 170 & $0.007(4)$ \\
\hline 69 & 157 & $0.06(2)$ \\
\hline & 158 & $0.126(25)$ \\
\hline & 159 & $0.227(23)$ \\
\hline & 160 & $0.273(54)$ \\
\hline & 161 & $0.294(30)$ \\
\hline
\end{tabular}




\begin{tabular}{|c|c|c|}
\hline \multicolumn{2}{|r|}{162} & $0.282(48)$ \\
\hline & 163 & $0.242(36)$ \\
\hline & 164 & $0.203(30)$ \\
\hline & 165 & $0.154(15)$ \\
\hline & 166 & $0.117(23)$ \\
\hline & 167 & $0.072(25)$ \\
\hline & 168 & $0.043(13)$ \\
\hline & 169 & $0.023(10)$ \\
\hline & 170 & $0.012(6)$ \\
\hline 70 & 159 & $0.076(39)$ \\
\hline & 160 & $0.150(38)$ \\
\hline & 161 & $0.220(22)$ \\
\hline & 162 & $0.248(38)$ \\
\hline & 163 & $0.266(27)$ \\
\hline & 164 & $0.254(39)$ \\
\hline & 165 & $0.251(38)$ \\
\hline & 166 & $0.220(34)$ \\
\hline & 167 & $0.170(21)$ \\
\hline & 168 & $0.143(22)$ \\
\hline & 169 & $0.093(14)$ \\
\hline & 170 & $0.066(7)$ \\
\hline & 171 & $0.040(8)$ \\
\hline & 172 & $0.022(7)$ \\
\hline & 173 & $0.015(6)$ \\
\hline & 174 & $0.005(3)$ \\
\hline 71 & 162 & $0.076(38)$ \\
\hline & 163 & $0.144(43)$ \\
\hline & 164 & $0.224(34)$ \\
\hline & 165 & $0.249(25)$ \\
\hline & 166 & $0.235(47)$ \\
\hline
\end{tabular}




\begin{tabular}{|c|c|c|}
\hline \multicolumn{2}{|r|}{67} & $0.218(22)$ \\
\hline & 168 & $0.194(29)$ \\
\hline & 169 & $0.166(20)$ \\
\hline & 170 & $0.125(13)$ \\
\hline & 171 & $0.0941(94)$ \\
\hline & 172 & $0.0623(93)$ \\
\hline & 173 & $0.0355(71)$ \\
\hline & 174 & $0.018(5)$ \\
\hline & 175 & $0.009(5)$ \\
\hline & 176 & $0.005(3)$ \\
\hline 72 & 164 & $0.075(23)$ \\
\hline & 165 & $0.148(30)$ \\
\hline & 166 & $0.202(30)$ \\
\hline & 167 & $0.235(23)$ \\
\hline & 168 & $0.259(26)$ \\
\hline & 169 & $0.231(35)$ \\
\hline & 170 & $0.200(30)$ \\
\hline & 171 & $0.174(26)$ \\
\hline & 172 & $0.140(28)$ \\
\hline & 173 & $0.095(19)$ \\
\hline & 174 & $0.061(12)$ \\
\hline & 175 & $0.040(10)$ \\
\hline & 176 & $0.022(8)$ \\
\hline & 177 & $0.013(7)$ \\
\hline & 178 & $0.006(4)$ \\
\hline 73 & 166 & $0.019(12)$ \\
\hline & 167 & $0.048(14)$ \\
\hline & 168 & $0.094(27)$ \\
\hline & 169 & $0.158(39)$ \\
\hline & 170 & $0.212(52)$ \\
\hline
\end{tabular}




\begin{tabular}{|c|c|c|}
\hline \multicolumn{2}{|r|}{171} & $0.257(77)$ \\
\hline & 172 & $0.228(66)$ \\
\hline & 173 & $0.194(50)$ \\
\hline & 174 & $0.162(49)$ \\
\hline & 175 & $0.098(30)$ \\
\hline & 176 & $0.064(24)$ \\
\hline & 177 & $0.034(17)$ \\
\hline & 178 & $0.018(9)$ \\
\hline & 179 & $0.010(6)$ \\
\hline & 180 & $0.006(4)$ \\
\hline 74 & 170 & $0.052(18)$ \\
\hline & 171 & $0.097(29)$ \\
\hline & 172 & $0.138(27)$ \\
\hline & 173 & $0.172(26)$ \\
\hline & 174 & $0.195(29)$ \\
\hline & 175 & $0.181(36)$ \\
\hline & 176 & $0.143(31)$ \\
\hline & 177 & $0.109(34)$ \\
\hline & 178 & $0.072(23)$ \\
\hline & 179 & $0.044(18)$ \\
\hline & 180 & $0.024(12)$ \\
\hline & 181 & $0.011(8)$ \\
\hline & 182 & $0.006(5)$ \\
\hline 75 & 173 & $0.03(2)$ \\
\hline & 174 & $0.07(3)$ \\
\hline & 175 & $0.11(4)$ \\
\hline & 176 & $0.15(3)$ \\
\hline & 177 & $0.18(4)$ \\
\hline & 178 & $0.18(4)$ \\
\hline & 179 & $0.15(6)$ \\
\hline
\end{tabular}




$\left|\begin{array}{c|c|}180 & 0.11(5) \\ 181 & 0.067(40) \\ 182 & 0.044(31) \\ 183 & 0.025(17) \\ 184 & 0.013(9)\end{array}\right|$

Table 2: Fission fragment isotopic cross sections measured in the present work. Statistical uncertainties are given on the last significant numbers, excluding the $10 \%$ systematical uncertainties. 